\title{
EXISTENCE AND MULTIPLICITY OF WEAK SOLUTIONS FOR A CLASS OF DEGENERATE NONLINEAR ELLIPTIC EQUATIONS
}

\author{
MIHAI MIHĂILESCU
}

Received 11 January 2005; Revised 4 July 2005; Accepted 17 July 2005

The goal of this paper is to study the existence and the multiplicity of non-trivial weak solutions for some degenerate nonlinear elliptic equations on the whole space $\mathbf{R}^{N}$. The solutions will be obtained in a subspace of the Sobolev space $W^{1, p}\left(\mathbf{R}^{N}\right)$. The proofs rely essentially on the Mountain Pass theorem and on Ekeland's Variational principle.

Copyright (c) 2006 Mihai Mihăilescu. This is an open access article distributed under the Creative Commons Attribution License, which permits unrestricted use, distribution, and reproduction in any medium, provided the original work is properly cited.

\section{Introduction}

The goal of this paper is to study a nonlinear elliptic equation in which the divergence form operator $-\operatorname{div}(a(x, \nabla u))$ is involved. Such operators appear in many nonlinear diffusion problems, in particular in the mathematical modeling of non-Newtonian fluids (see [5] for a discussion of some physical background). Particularly, the $p$-Laplacian operator $-\operatorname{div}\left(|\nabla u|^{p-2} \nabla u\right)$ is a special case of the operator $-\operatorname{div}(a(x, \nabla u))$. Problems involving the $p$-Laplacian operator have been intensively studied in the last decades. We just remember the work on that topic of João Marcos B. do Ó [7], Pflüger [12], Rădulescu and Smets [14] and the references therein. In the case of more general types of operators we point out the papers of João Marcos B. do Ó [6] and Nápoli and Mariani [4]. On the other hand, when the operator $-\operatorname{div}(a(x, \nabla u))$ is of degenerate type we refer to Cîrstea and Rădulescu [15] and Motreanu and Rădulescu [11].

In this paper we study the existence and multiplicity of non-trivial weak solutions to equations of the type

$$
-\operatorname{div}(a(x, \nabla u))=\mathscr{F}(x, u), \quad x \in \mathbf{R}^{N},
$$

where the operator $\operatorname{div}(a(x, \nabla u))$ is nonlinear (and can be also degenerate), $N \geq 3$ and function $\mathscr{F}(x, u)$ satisfies several hypotheses. Our goal is to show how variational techniques based on the Mountain Pass theorem (see Ambrosetti and Rabinowitz [2]) and Ekeland's Variational principle (see Ekeland [8]) can be used in order to get existence of 
one or two solutions for equations of type (1.1). Results regarding the multiplicity of solutions have been originally proven by Tarantello [16], but in the case of linear equations and in a different framework. More precisely, Tarantello proved that the equation

$$
-\Delta u=|u|^{4 /(N-2)} u+\Gamma(x)
$$

has at least two distinct solutions, in a bounded domain of $\mathbf{R}^{N}(N \geq 3)$, provided that $\Gamma \not \equiv 0$ is sufficiently "small" in a suitable sense.

\section{Main results}

The starting point of our discussion is the equation

$$
-\Delta v+b(x) v=f(x, v) \quad x \in \mathbf{R}^{N}
$$

studied by Rabinowitz in [13]. Assuming that function $f(x, v)$ is subcritical and satisfies a condition of the Ambrosetti-Rabinowitz type (see [2]) and function $b(x)$ is sufficiently smooth and unbounded at infinity, it is showed in [13] that problem (2.1) has a nontrivial weak solution in the classical Sobolev space $W^{1,2}\left(\mathbf{R}^{N}\right)$.

In the case when $b(x)$ is continuous and nonnegative and $f(x, v)=h(x) v^{\alpha}+v^{\beta}$ is such that $h: \mathbf{R}^{N} \rightarrow \mathbf{R}$ is some integrable function and $1<\alpha<2<\beta<(N+2) /(N-2), N \geq 3$, Gonçalves and Miyagaki proved in [9] that problem (2.1) has at least two nonnegative solutions in a subspace of $W^{1,2}\left(\mathbf{R}^{N}\right)$. In a similar framework, when $f(x, v)=\lambda v^{\alpha}+v^{2^{\star}-1}$ with $0<\alpha<1$ and $2^{\star}=(2 N) /(N-2), N \geq 3$ it is shown in [1] that problem (2.1) has a nonnegative solution for $\lambda$ positive and small enough. Furthermore, in [1] it is also proved that in the case $N \geq 4$ and $\alpha=1$ problem (2.1) has a nonnegative solution provided that $\lambda$ is positive and small enough. For more information and connections on (2.1) the reader may consult the references in [9].

In this paper our aim is to study the problem

$$
-\operatorname{div}(a(x, \nabla u))+b(x)|u|^{p-2} u=f(x, u), \quad x \in \mathbf{R}^{N},
$$

where $N \geq 3$ and $2 \leq p<N$.

We point out the fact that in the case when $a(x, \nabla u)=|x|^{\alpha} \nabla u, \alpha \in(0,2)$ and $p=2$ problem (2.2) was studied by Mihăilescu and Rădulescu in [10]. In that paper the authors present the connections between such equations and some Schrödinger equations with Hardy potential and show that (2.2) has a nontrivial weak solution. A discussion of some physical applications for equations of type (2.2) and a list of papers devoted with the study of such problems is also included in [10].

In the following we describe the framework in which we will study (2.2).

Consider $a: \mathbf{R}^{N} \times \mathbf{R}^{N} \rightarrow \mathbf{R}^{N}, a=a(x, \xi)$, is the continuous derivative with respect to $\xi$ of the continuous function $A: \mathbf{R}^{N} \times \mathbf{R}^{N} \rightarrow \mathbf{R}, A=A(x, \xi)$, that is, $a(x, \xi)=(d / d \xi) A(x, \xi)$. 
Suppose that $a$ and $A$ satisfy the hypotheses below:

(A1) $A(x, 0)=0$ for all $x \in \mathbf{R}^{N}$;

(A2) $|a(x, \xi)| \leq c_{1}\left(\theta(x)+|\xi|^{p-1}\right)$, for all $x, \xi \in \mathbf{R}^{N}$, with $c_{1}$ a positive constant and $\theta: \mathbf{R}^{N} \rightarrow \mathbf{R}$ is a function such that $\theta(x) \geq 0$ for all $x \in \mathbf{R}^{N}$ and $\theta \in L^{\infty}\left(\mathbf{R}^{N}\right) \cap$ $L^{p /(p-1)}\left(\mathbf{R}^{N}\right)$

(A3) there exists $k>0$ such that

$$
A\left(x, \frac{\xi+\psi}{2}\right) \leq \frac{1}{2} A(x, \xi)+\frac{1}{2} A(x, \psi)-k|\xi-\psi|^{p}
$$

for all $x, \xi, \psi \in \mathbf{R}^{N}$, that is, $A(x, \cdot)$ is $p$-uniformly convex;

(A4) $0 \leq a(x, \xi) \cdot \xi \leq p A(x, \xi)$, for all $x, \xi \in \mathbf{R}^{N}$;

(A5) there exists a constant $\Lambda>0$ such that

$$
A(x, \xi) \geq \Lambda|\xi|^{p}
$$

for all $x, \xi \in \mathbf{R}^{N}$.

Examples. (1) $A(x, \xi)=(1 / p)|\xi|^{p}, a(x, \xi)=|\xi|^{p-2} \xi$, with $p \geq 2$ and we get the $p$-Laplacian operator

$$
\operatorname{div}\left(|\nabla u|^{p-2} \nabla u\right)
$$

(2) $A(x, \xi)=(1 / p)|\xi|^{p}+\theta(x)\left[\left(1+|\xi|^{2}\right)^{1 / 2}-1\right], a(x, \xi)=|\xi|^{p-2} \xi+\theta(x)\left(\xi /\left(1+|\xi|^{2}\right)^{1 / 2}\right)$, with $p \geq 2$ and $\theta$ a function which verifies the conditions from (A2). We get the operator

$$
\operatorname{div}\left(|\nabla u|^{p-2} \nabla u\right)+\operatorname{div}\left(\theta(x) \frac{\nabla u}{\left(1+|\nabla u|^{2}\right)^{1 / 2}}\right)
$$

which can be regarded as the sum between the $p$-Laplacian operator and a degenerate form of the mean curvature operator.

(3) $A(x, \xi)=(1 / p)\left[\left(\theta(x)^{2 /(p-1)}+|\xi|^{2}\right)^{p / 2}-\theta(x)^{p /(p-1)}\right], \quad a(x, \xi)=\left(\theta(x)^{2 /(p-1)}+\right.$ $\left.|\xi|^{2}\right)^{(p-2) / 2} \xi$, with $p \geq 2$ and $\theta$ a function which verifies the conditions from (A2). We get the operator

$$
\operatorname{div}\left(\left(\theta(x)^{2 /(p-1)}+|\nabla u|^{2}\right)^{(p-2) / 2} \nabla u\right)
$$

which is a variant of the generalized mean curvature operator, $\operatorname{div}\left(\left(1+|\nabla u|^{2}\right)^{(p-2) / 2} \nabla u\right)$.

Assume that function $b: \mathbf{R}^{N} \rightarrow \mathbf{R}$ is continuous and verifies the hypotheses:

(B) There exists a positive constant $b_{0}>0$ such that

$$
b(x) \geq b_{0}>0
$$

for all $x \in \mathbf{R}^{N}$.

In a first instance we assume that function $f: \mathbf{R}^{N} \times \mathbf{R} \rightarrow \mathbf{R}$ satisfies the hypotheses:

(F1) $f \in C^{1}\left(\mathbf{R}^{N} \times \mathbf{R}, \mathbf{R}\right), f=f(x, z)$ and $f(x, 0)=0$ for all $x \in \mathbf{R}^{N}$; 
(F2) there exist two functions $\tau_{1}, \tau_{2}: \mathbf{R}^{N} \rightarrow \mathbf{R}, \tau_{1}(x), \tau_{2}(x) \geq 0$ for a.e. $x \in \mathbf{R}^{N}$ and two constants $r, s \in(p-1,(N p-N+p) /(N-p))$ such that

$$
\left|f_{z}(x, z)\right| \leq \tau_{1}(x)|z|^{r-1}+\tau_{2}(x)|z|^{s-1},
$$

for all $x \in \mathbf{R}^{N}$ and all $z \in \mathbf{R}$, where $\tau_{1} \in L^{r_{0}}\left(\mathbf{R}^{N}\right) \cap L^{\infty}\left(\mathbf{R}^{N}\right), \tau_{2} \in L^{s_{0}}\left(\mathbf{R}^{N}\right) \cap$ $L^{\infty}\left(\mathbf{R}^{N}\right)$, with $r_{0}=N p /(N p-(r+1)(N-p))$ and $s_{0}=N p /(N p-(s+1)(N-p))$;

(F3) there exists a constant $\mu>p$ such that

$$
0<\mu F(x, z):=\mu \int_{0}^{z} f(x, t) d t \leq z f(x, z)
$$

for all $x \in \mathbf{R}^{N}$ and all $z \in \mathbf{R} \backslash\{0\}$.

Next, we study the problem

$$
-\operatorname{div}(a(x, \nabla u))+b(x)|u|^{p-2} u=h(x)|u|^{q-1} u+g(x)|u|^{s-1} u, \quad x \in \mathbf{R}^{N}
$$

with $1<q<p-1<s<(N p-N+p) /(N-p)$ and $N \geq 3$.

Our basic assumptions on functions $h$ and $g: \mathbf{R}^{N} \rightarrow \mathbf{R}$ are the following:

(H) $h(x) \geq 0$ for all $x \in \mathbf{R}^{N}$ and $h \in L^{q_{0}}\left(\mathbf{R}^{N}\right) \cap L^{\infty}\left(\mathbf{R}^{N}\right)$, where $q_{0}=N p /(N p-(q+$ 1) $(N-p))$;

(G) $g(x) \geq 0$ for all $x \in \mathbf{R}^{N}$ and $g \in L^{s_{0}}\left(\mathbf{R}^{N}\right) \cap L^{\infty}\left(\mathbf{R}^{N}\right)$, where $s_{0}=N p /(N p-(s+$ 1) $(N-p))$.

Let $W^{1, p}\left(\mathbf{R}^{N}\right)$ be the usual Sobolev space under the norm

$$
\|u\|_{1}=\left(\int_{\mathbf{R}^{N}}\left(|\nabla u|^{p}+|u|^{p}\right) d x\right)^{1 / p}
$$

and consider the subspace of $W^{1, p}\left(\mathbf{R}^{N}\right)$

$$
E=\left\{u \in W^{1, p}\left(\mathbf{R}^{N}\right) ; \int_{\mathbf{R}^{N}}\left(|\nabla u|^{p}+b(x)|u|^{p}\right) d x<\infty\right\} .
$$

The Banach space $E$ can be endowed with the norm

$$
\|u\|^{p}=\int_{\mathbf{R}^{N}}\left(|\nabla u|^{p}+b(x)|u|^{p}\right) d x .
$$

Moreover,

$$
\|u\| \geq m_{0}^{1 / p}\|u\|_{1}
$$

with $m_{0}=\min \left\{1, b_{0}\right\}$. Thus the continuous embeddings

$$
E \hookrightarrow W^{1, p}\left(\mathbf{R}^{N}\right) \hookrightarrow L^{i}\left(\mathbf{R}^{N}\right), \quad p \leq i \leq p^{\star}, p^{\star}=\frac{N p}{N-p}
$$

hold true. 
We say that $u \in E$ is a weak solution for problem (2.2) if

$$
\int_{\mathbf{R}^{N}} a(x, \nabla u) \cdot \nabla \varphi d x+\int_{\mathbf{R}^{N}} b(x)|u|^{p-2} u \varphi d x-\int_{\mathbf{R}^{N}} f(x, u) \varphi d x=0,
$$

for all $\varphi \in E$.

Similarly, we say that $u \in E$ is a weak solution for problem (2.11) if

$$
\begin{aligned}
\int_{\mathbf{R}^{N}} a(x, \nabla u) \cdot \nabla \varphi d x+\int_{\mathbf{R}^{N}} b(x)|u|^{p-2} u \varphi d x \\
-\int_{\mathbf{R}^{N}} h(x)|u|^{q-1} u \varphi d x-\int_{\mathbf{R}^{N}} g(x)|u|^{s-1} u \varphi d x=0,
\end{aligned}
$$

for all $\varphi \in E$.

Our main results are given by the following two theorems.

Theorem 2.1. Assuming hypotheses (A1)-(A5), (B) and (F1)-(F3) are fulfilled then problem (2.2) has at least one non-trivial weak solution.

Theorem 2.2. Assume $1<q<p-1<s<(N p-N+p) /(N-p)$ and conditions (A1)(A5), (B), (H) and $(G)$ are fulfilled. Then problem (2.11) has at least two non-trivial weak solutions provided that the product $\|h\|_{L^{q_{0}}\left(\mathbf{R}^{N}\right)}^{(s+1-p) /(s-q)} \cdot\|g\|_{L^{s_{0}\left(\mathbf{R}^{N}\right)}}^{(p-q-1) /(s-q)}$ is small enough.

\section{Auxiliary results}

In this section we study certain properties of functional $T: E \rightarrow \mathbf{R}$ defined by

$$
T(u)=\int_{\mathbf{R}^{N}} A(x, \nabla u) d x+\frac{1}{p} \int_{\mathbf{R}^{N}} b(x)|u|^{p} d x,
$$

for all $u \in E$. It is easy to remark that $T \in C^{1}(E, \mathbf{R})$ and

$$
\left\langle T^{\prime}(u), v\right\rangle=\int_{\mathbf{R}^{N}} a(x, \nabla u) \cdot \nabla v d x+\int_{\mathbf{R}^{N}} b(x)|u|^{p-2} u v d x
$$

for all $u, v \in E$.

Proposition 3.1. Functional $T$ is weakly lower semicontinuous.

Proof. Let $u \in E$ and $\epsilon>0$ be fixed. Using the properties of lower semicontinuous functions (see [3, Section I.3]) is enough to prove that there exists $\delta>0$ such that

$$
T(v) \geq T(u)-\epsilon, \quad \forall v \in E \text { with }\|u-v\|<\delta .
$$

We remember Clarkson's inequality (see [3, page 59])

$$
\left|\frac{\alpha+\beta}{2}\right|^{p}+\left|\frac{\alpha-\beta}{2}\right|^{p} \leq \frac{1}{2}\left(|\alpha|^{p}+|\beta|^{p}\right), \quad \forall \alpha, \beta \in \mathbf{R} .
$$


Thus we deduce that

$$
\begin{aligned}
\int_{\mathbf{R}^{N}} b(x)\left|\frac{u+v}{2}\right|^{p} d x+\int_{\mathbf{R}^{N}} b(x)\left|\frac{u-v}{2}\right|^{p} d x \\
\leq \frac{1}{2} \int_{\mathbf{R}^{N}} b(x)|u|^{p} d x+\frac{1}{2} \int_{\mathbf{R}^{N}} b(x)|v|^{p} d x, \quad \forall u, v \in E .
\end{aligned}
$$

The above inequality and condition (A3) imply that there exists a positive constant $k_{1}>0$ such that

$$
T\left(\frac{u+v}{2}\right) \leq \frac{1}{2} T(u)+\frac{1}{2} T(v)-k_{1}\|u-v\|^{p}, \quad \forall u, v \in E,
$$

that is, $T$ is $p$-uniformly convex.

Since $T$ is convex we have

$$
T(v) \geq T(u)+\left\langle T^{\prime}(u), v-u\right\rangle, \quad \forall v \in E .
$$

Using condition (A2) and Hölder's inequality we deduce that there exists a positive constant $C>0$ such that

$$
\begin{aligned}
T(v) \geq & T(u)-\int_{\mathbf{R}^{N}}|a(x, \nabla u)| \cdot|\nabla v-\nabla u| d x-\int_{\mathbf{R}^{N}} b(x)|u|^{p-1}|u-v| d x \\
\geq & T(u)-\int_{\mathbf{R}^{N}} c_{1}\left(\theta(x)+|\nabla u|^{p-1}\right)|\nabla v-\nabla u| d x \\
& -\int_{\mathbf{R}^{N}} b(x)^{(p-1) / p}|u|^{p-1} b(x)^{1 / p}|u-v| d x \\
\geq & T(u)-c_{1} \cdot\left(\|\theta\|_{L^{p /(p-1)}\left(\mathbf{R}^{N}\right)}+\|\nabla u\|_{L^{p}\left(\mathbf{R}^{N}\right)}^{p-1}\right) \cdot\left(\int_{\mathbf{R}^{N}}|\nabla v-\nabla u|^{p} d x\right)^{1 / p} \\
& -\left(\int_{\mathbf{R}^{N}} b(x)|u|^{p} d x\right)^{(p-1) / p} \cdot\left(\int_{\mathbf{R}^{N}} b(x)|v-u|^{p} d x\right)^{1 / p} \\
\geq & T(u)-C\|u-v\|, \quad \forall v \in E .
\end{aligned}
$$

It is clear that taking $\delta=\epsilon / C$ relation (3.3) holds true for all $v \in E$ with $\|v-u\|<\delta$. Thus we have proved that $T$ is strongly lower semicontinuous. Taking into account the fact that $T$ is convex then by [3, Corollary III.8] we conclude that $T$ is weakly lower semicontinuous and the proof of Proposition 3.1 is complete.

Proposition 3.2. Assume $\left\{u_{n}\right\}$ is a subsequence from $E$ which is weakly convergent to $u \in E$ and

$$
\limsup _{n \rightarrow \infty}\left\langle T^{\prime}\left(u_{n}\right), u_{n}-u\right\rangle \leq 0
$$

Then $\left\{u_{n}\right\}$ converges strongly to $u$ in $E$.

Proof. Since $\left\{u_{n}\right\}$ is weakly convergent to $u$ in $E$ it follows that $\left\{u_{n}\right\}$ is bounded in $E$. 
By conditions (A2) and (A3) we have

$$
\begin{aligned}
0 \leq A(x, \xi) & =\int_{0}^{1} \frac{d}{d t} A(x, t \xi) d t=\int_{0}^{1} a(x, t \xi) \cdot \xi d t \\
& \leq c_{1} \int_{0}^{1}\left(\theta(x)+|\xi|^{p-1} t^{p-1}\right) d t \\
& \leq c_{1}\left(\theta(x)|\xi|+\frac{1}{p}|\xi|^{p}\right), \quad \forall x, \xi \in \mathbf{R}^{N}
\end{aligned}
$$

Thus, there exists a constant $c_{2}>0$ such that

$$
|A(x, \xi)| \leq c_{2}\left(\theta(x)|\xi|+|\xi|^{p}\right), \quad \forall x, \xi \in \mathbf{R}^{N}
$$

Relation (3.11) and Hölder's inequality imply

$$
\begin{aligned}
\int_{\mathbf{R}^{N}} A\left(x, \nabla u_{n}\right) d x & \leq c_{2}\left(\int_{\mathbf{R}^{N}} \theta(x)\left|\nabla u_{n}\right| d x+\int_{\mathbf{R}^{N}}\left|\nabla u_{n}\right|^{p} d x\right) \\
& \leq c_{2} \cdot\left(\|\theta\|_{L^{p /(p-1)}\left(\mathbf{R}^{N}\right)} \cdot\left\|u_{n}\right\|+\left\|u_{n}\right\|^{p}\right) .
\end{aligned}
$$

The above inequality and the fact that $\left\{u_{n}\right\}$ is bounded in $E$ show that there exists $M_{1}>0$ such that $T\left(u_{n}\right) \leq M_{1}$ for all $n$. Then we may assume that $T\left(u_{n}\right) \rightarrow \gamma$. Using Proposition 3.1 we find

$$
T(u) \leq \liminf _{n \rightarrow \infty} T\left(u_{n}\right)=\gamma
$$

Since $T$ is convex the following inequality holds true

$$
T(u) \geq T\left(u_{n}\right)+\left\langle T^{\prime}\left(u_{n}\right), u_{n}-u\right\rangle, \quad \forall n .
$$

Relation (3.9) and the above inequality imply $T(u) \geq \gamma$ and thus $T(u)=\gamma$.

We also have $\left(u_{n}+u\right) / 2$ converges weakly to $u$ in $E$. Using again Proposition 3.1 we deduce

$$
\gamma=T(u) \leq \liminf _{n \rightarrow \infty} T\left(\frac{u_{n}+u}{2}\right)
$$

If we assume by contradiction that $\left\|u_{n}-u\right\|$ does not converge to 0 then there exists $\epsilon>0$ such that passing to a subsequence $\left\{u_{n m}\right\}$ we have $\left\|u_{n m}-u\right\| \geq \epsilon$. That fact and relation (3.6) imply

$$
\frac{1}{2} T(u)+\frac{1}{2} T\left(u_{n m}\right)-T\left(\frac{u+u_{n m}}{2}\right) \geq k_{1}\left\|u-u_{n m}\right\|^{p} \geq k_{1} \epsilon^{p}
$$

Letting $m \rightarrow \infty$ we find

$$
\limsup _{m \rightarrow \infty} T\left(\frac{u+u_{n m}}{2}\right) \leq \gamma-k_{1} \epsilon^{p}
$$


and that is a contradiction with (3.15). Thus we have

$$
\left\|u_{n}-u\right\| \longrightarrow 0
$$

The proof of Proposition 3.2 is complete.

\section{Proof of Theorem 2.1}

In order to prove Theorem 2.1 we define the functional

$$
J(u)=\int_{\mathbf{R}^{N}} A(x, \nabla u) d x+\frac{1}{p} \int_{\mathbf{R}^{N}} b(x)|u|^{p} d x-\int_{\mathbf{R}^{N}} F(x, u) d x .
$$

$J: E \rightarrow \mathbf{R}$ is well defined and of class $C^{1}$ with the derivative given by

$$
\left\langle J^{\prime}(u), \varphi\right\rangle=\int_{\mathbf{R}^{N}} a(x, \nabla u) \cdot \nabla \varphi d x+\int_{\mathbf{R}^{N}} b(x)|u|^{p-2} u \varphi d x-\int_{\mathbf{R}^{N}} f(x, u) \varphi d x,
$$

for all $u, \varphi \in E$. We have denoted by $\langle$,$\rangle the duality pairing between E$ and $E^{\star}$, where $E^{\star}$ is the dual of $E$.

We remark that the critical points of the functional $J$ correspond to the weak solutions of (2.2). Thus, our idea is to apply the Mountain Pass theorem (see [2]) in order to obtain a non-trivial critical point and thus a non-trivial weak solution.

First, we prove a lemma which shows that functional $J$ has a mountain-pass geometry.

Lemma 4.1. (1) There exist $\rho>0$ and $\varrho>0$ such that

$$
J(u) \geq \varrho>0, \quad \forall u \in E \text { with }\|u\|=\rho .
$$

(2) There exists $u_{0} \in E$ such that

$$
\lim _{t \rightarrow \infty} J\left(t u_{0}\right)=-\infty
$$

Proof. (1) By (F2) there exist $A_{1}, A_{2}>0$ two constants such that

$$
0 \leq F(x, z) \leq A_{1}|z|^{r+1}+A_{2}|z|^{s+1} .
$$

Then we deduce that

$$
\lim _{|z| \rightarrow 0} \frac{F(x, z)}{|z|^{p}}=0, \quad \lim _{|z| \rightarrow \infty} \frac{F(x, z)}{|z|^{p^{\star}}}=0
$$

Then, for a $\epsilon>0$ there exist two constants $\delta_{1}$ and $\delta_{2}$ such that

$$
\begin{array}{ll}
F(x, z)<\epsilon|z|^{p} & \forall z \text { with }|z|<\delta_{1}, \\
F(x, z)<\epsilon|z|^{p^{\star}} \quad \forall z \text { with }|z|>\delta_{2} .
\end{array}
$$

Relation (4.5) implies that for all $z$ with $|z| \in\left[\delta_{1}, \delta_{2}\right]$ there exists a positive constant $C>0$ such that

$$
F(x, z)<C
$$


We obtain that for all $\epsilon>0$ there exists $C_{\epsilon}>0$ such that

$$
F(x, z) \leq \epsilon|z|^{p}+C_{\epsilon}|z|^{p^{\star}} .
$$

Relation (4.9), conditions (A5) and (b1) and the Sobolev embedding imply

$$
\begin{aligned}
J(u) & =\int_{\mathbf{R}^{N}} A(x, \nabla u) d x+\frac{1}{p} \int_{\mathbf{R}^{N}} b(x)|u|^{p} d x-\int_{\mathbf{R}^{N}} F(x, u) d x \\
& \geq \Lambda \int_{\mathbf{R}^{N}}|\nabla u|^{p} d x+\frac{1}{p} \int_{\mathbf{R}^{N}} b(x)|u|^{p} d x-\epsilon \int_{\mathbf{R}^{N}}|u|^{p} d x-C_{\epsilon} \int_{\mathbf{R}^{N}}|u|^{p^{\star}} d x \\
& \geq \min \left\{\Lambda, \frac{1}{p}\right\} \cdot\|u\|^{p}-\frac{\epsilon}{b_{0}} \int_{\mathbf{R}^{N}} b(x)|u|^{p} d x-C_{\epsilon} \int_{\mathbf{R}^{N}}|u|^{p^{\star}} d x \\
& \geq\|u\|^{p} \cdot\left[\left(\min \left\{\Lambda, \frac{1}{p}\right\}-\frac{\epsilon}{b_{0}}\right)-C_{\epsilon}^{\prime} \cdot\|u\|^{p^{\star}-p}\right] .
\end{aligned}
$$

Letting $\epsilon \in\left(0, \min \{\Lambda, 1 / p\} \cdot b_{0}\right)$ be fixed, we obtain that the first part of Lemma 4.1 holds true.

(2) To prove the second part of the lemma, first, we remark that by condition (F3) we have

$$
F(x, z) \geq \lambda|z|^{\mu}, \quad \forall|z| \geq \eta, x \in \mathbf{R}^{N},
$$

where $\lambda$ and $\eta$ are two positive constants.

On the other hand we claim that

$$
A(x, z \xi) \leq A(x, \xi) z^{p}, \quad \forall z \geq 1, x, \xi \in \mathbf{R}^{N} .
$$

Indeed, if we put $\alpha(t)=A(x, t \xi)$ then by (A1) and (A4) we have

$$
\alpha^{\prime}(t)=a(x, t \xi) \cdot \xi=\frac{1}{t} a(x, t \xi) \cdot(t \xi) \leq \frac{p}{t} A(x, t \xi)=\frac{p}{t} \alpha(t) .
$$

Hence

$$
\frac{\alpha^{\prime}(t)}{\alpha(t)} \leq \frac{p}{t}
$$

or

$$
\log (\alpha(t))-\log (\alpha(1)) \leq p \log (t)
$$

We deduce that $\alpha(t) / \alpha(1) \leq t^{p}$ and thus (4.12) holds true. 
Let now $u_{0} \in E$ be such that meas $\left(\left\{x \in \mathbf{R}^{N} ;\left|u_{0}(x)\right| \geq \eta\right\}\right)>0$. Using relations (4.11) and (4.12) we obtain

$$
\begin{aligned}
J\left(t u_{0}\right)= & \int_{\mathbf{R}^{N}}\left[A\left(x, t \nabla u_{0}\right)+\frac{1}{p} b(x) t^{p}\left|u_{0}\right|^{p}\right] d x-\int_{\mathbf{R}^{N}} F\left(x, t u_{0}\right) d x \\
\leq & t^{p} \int_{\mathbf{R}^{N}}\left[A\left(x, \nabla u_{0}\right)+\frac{1}{p} b(x)\left|u_{0}\right|^{p}\right] d x-\int_{\left\{x \in \mathbf{R}^{N} ;\left|u_{0}(x)\right| \geq \eta\right\}} F\left(x, t u_{0}\right) d x \\
& -\int_{\left\{x \in \mathbf{R}^{N} ;\left|u_{0}(x)\right| \leq \eta\right\}} F\left(x, t u_{0}\right) d x \\
\leq & t^{p} \int_{\mathbf{R}^{N}}\left[A\left(x, \nabla u_{0}\right)+\frac{1}{p} b(x)\left|u_{0}\right|^{p}\right] d x-t^{\mu} \lambda \int_{\left\{x \in \mathbf{R}^{N} ;\left|u_{0}(x)\right| \geq \eta\right\}}\left|u_{0}\right|^{\mu} d x .
\end{aligned}
$$

Since $\mu>p$ the right-hand side of the above inequality converges to $-\infty$ as $t \rightarrow \infty$.

The lemma is completely proved.

Proof of Theorem 2.1. Using Lemma 4.1 we may apply the Mountain Pass theorem (see [2]) to functional $J$. We obtain that there exists a sequence $\left\{u_{n}\right\}$ in $E$ such that

$$
J\left(u_{n}\right) \longrightarrow c>0, \quad J^{\prime}\left(u_{n}\right) \longrightarrow 0 \quad \text { in } E^{\star}
$$

We prove that $\left\{u_{n}\right\}$ is bounded in $E$. We assume by contradiction that $\left\|u_{n}\right\| \rightarrow \infty$ as $n \rightarrow$ $\infty$. Then, using relation (4.17) and conditions (A4), (A5) and (F3) we deduce that for $n$ large enough the following inequalities hold

$$
\begin{aligned}
c+1+\left\|u_{n}\right\| \geq & J\left(u_{n}\right)-\frac{1}{\mu}\left\langle J^{\prime}\left(u_{n}\right), u_{n}\right\rangle \\
= & \int_{\mathbf{R}^{N}}\left[A\left(x, \nabla u_{n}\right)-\frac{1}{\mu} a\left(x, \nabla u_{n}\right) \cdot \nabla u_{n}\right] d x \\
& +\int_{\mathbf{R}^{N}}\left[\frac{1}{p} b(x)\left|u_{n}\right|^{p}-\frac{1}{\mu} b(x)\left|u_{n}\right|^{p}\right] d x \\
& +\int_{\mathbf{R}^{N}}\left[\frac{1}{\mu} f\left(x, u_{n}\right) u_{n}-F\left(x, u_{n}\right)\right] d x \\
\geq & \left(1-\frac{p}{\mu}\right) \int_{\mathbf{R}^{N}} A\left(x, \nabla u_{n}\right) d x+\left(\frac{1}{p}-\frac{1}{\mu}\right) \int_{\mathbf{R}^{N}} b(x)\left|u_{n}\right|^{p} d x \\
\geq & \left(1-\frac{p}{\mu}\right) \Lambda \int_{\mathbf{R}^{N}}\left|\nabla u_{n}\right|^{p} d x+\left(\frac{1}{p}-\frac{1}{\mu}\right) \int_{\mathbf{R}^{N}} b(x)\left|u_{n}\right|^{p} d x \\
\geq & \min \left\{\left(1-\frac{p}{\mu}\right) \Lambda, \frac{1}{p}-\frac{1}{\mu}\right\} \cdot|| u_{n} \|^{p} .
\end{aligned}
$$

Dividing by $\left\|u_{n}\right\|$ and letting $n \rightarrow \infty$ we obtain a contradiction. Therefore $\left\{u_{n}\right\}$ is bounded in $E$ by a positive constant denoted by $M$. It follows that there exists $u \in E$ such that, passing to a subsequence still denoted by $\left\{u_{n}\right\}$, it converges weakly to $u$ in $E$ and $u_{n}(x) \rightarrow u(x)$ a.e. $x \in \mathbf{R}^{N}$. Since $E$ is continuously embedded in $L^{p^{*}}\left(\mathbf{R}^{N}\right)$ by [17, Theorem 10.36] we deduce that $u_{n}$ converges weakly to $u$ in $L^{p^{\star}}\left(\mathbf{R}^{N}\right)$. Then it is clear that $\left|u_{n}\right|^{r-1} u_{n}$ converges weakly to $|u|^{r-1} u$ in $L^{p^{\star} / r}\left(\mathbf{R}^{N}\right)$. 
Define the operator $U: L^{p^{\star} / r}\left(\mathbf{R}^{N}\right) \rightarrow \mathbf{R}$ by

$$
\langle U, w\rangle=\int_{\mathbf{R}^{N}} \tau_{1}(x) u w d x
$$

We remark that $U$ is linear and continuous provided that $\tau_{1} \in L^{r_{0}}\left(\mathbf{R}^{N}\right), u \in L^{p^{\star}}\left(\mathbf{R}^{N}\right)$ and $1 / p^{\star}+r / p^{\star}+1 / r_{0}=1$. All the above pieces of information imply

$$
\left\langle U,\left|u_{n}\right|^{r-1} u_{n}\right\rangle \rightarrow\left\langle U,|u|^{r-1} u\right\rangle
$$

that is,

$$
\lim _{n \rightarrow \infty} \int_{\mathbf{R}^{N}} \tau_{1}(x)\left|u_{n}\right|^{r-1} u_{n} u d x=\int_{\mathbf{R}^{N}} \tau_{1}(x)|u|^{r+1} d x
$$

With the same arguments we can show that

$$
\begin{gathered}
\lim _{n \rightarrow \infty} \int_{\mathbf{R}^{N}} \tau_{2}(x)\left|u_{n}\right|^{s-1} u_{n} u d x=\int_{\mathbf{R}^{N}} \tau_{2}(x)|u|^{s+1} d x, \\
\lim _{n \rightarrow \infty} \int_{\mathbf{R}^{N}} \tau_{1}(x)\left|u_{n}\right|^{r+1} d x=\int_{\mathbf{R}^{N}} \tau_{1}(x)|u|^{r+1} d x, \\
\lim _{n \rightarrow \infty} \int_{\mathbf{R}^{N}} \tau_{2}(x)\left|u_{n}\right|^{s+1} d x=\int_{\mathbf{R}^{N}} \tau_{2}(x)|u|^{s+1} d x .
\end{gathered}
$$

Relations (4.21), (4.23) and the fact that

$$
\begin{aligned}
\int_{\mathbf{R}^{N}} \tau_{1}(x)\left|u_{n}\right|^{r-1} u_{n}\left(u_{n}-u\right) d x= & \int_{\mathbf{R}^{N}} \tau_{1}(x)\left|u_{n}\right|^{r+1} d x-\int_{\mathbf{R}^{N}} \tau_{1}(x)|u|^{r+1} d x \\
& +\int_{\mathbf{R}^{N}} \tau_{1}(x)|u|^{r+1} d x-\int_{\mathbf{R}^{N}} \tau_{1}(x)\left|u_{n}\right|^{q-1} u_{n} u d x
\end{aligned}
$$

yield

$$
\lim _{n \rightarrow \infty} \int_{\mathbf{R}^{N}} \tau_{1}(x)\left|u_{n}\right|^{r-1} u_{n}\left(u_{n}-u\right) d x=0 .
$$

Similarly we obtain

$$
\lim _{n \rightarrow \infty} \int_{\mathbf{R}^{N}} \tau_{2}(x)\left|u_{n}\right|^{s-1} u_{n}\left(u_{n}-u\right) d x=0 .
$$

By (4.26), (4.27) and condition (F2) we get

$$
\lim _{n \rightarrow \infty} \int_{\mathbf{R}^{N}} f\left(x, u_{n}\right)\left(u_{n}-u\right) d x=0 .
$$

On the other hand we have

$$
\begin{gathered}
\int_{\mathbf{R}^{N}} a\left(x, \nabla u_{n}\right) \cdot \nabla u_{n} d x+\int_{\mathbf{R}^{N}} b(x)\left|u_{n}\right|^{p-2} u_{n}\left(u_{n}-u\right) d x \\
=\left\langle J^{\prime}\left(u_{n}\right), u_{n}-u\right\rangle+\int_{\mathbf{R}^{N}} f\left(x, u_{n}\right)\left(u_{n}-u\right) d x .
\end{gathered}
$$


Relations (4.28) and (4.29) imply

$$
\lim _{n \rightarrow \infty}\left(\int_{\mathbf{R}^{N}} a\left(x, \nabla u_{n}\right) \cdot \nabla\left(u_{n}-u\right) d x+\int_{\mathbf{R}^{N}} b(x)\left|u_{n}\right|^{p-2}\left(u_{n}-u\right) d x\right)=0,
$$

that is,

$$
\lim _{n \rightarrow \infty}\left\langle T^{\prime}\left(u_{n}\right), u_{n}-u\right\rangle=0
$$

where $T$ is the functional defined in the above section. Then applying Proposition 3.2 we deduce that $\left\{u_{n}\right\}$ converges strongly to $u$ in $E$. Since $J \in C^{1}(E, \mathbf{R})$ by $(4.17)$ we deduce that $\left\langle J^{\prime}(u), \varphi\right\rangle=0$ for all $\varphi \in E$, that is, $u$ is a weak solution of problem (2.2). Relation (4.17) also implies that $J(u)=c>0$ and that shows that $u$ is non-trivial.

The proof of Theorem 2.1 is complete.

\section{Proof of Theorem 2.2}

We remark that the weak solutions of (2.11) correspond to the critical points of the energy functional $I: E \rightarrow \mathbf{R}$ defined as follows

$$
\begin{aligned}
I(u)= & \int_{\mathbf{R}^{N}} A(x, \nabla u) d x+\frac{1}{p} \int_{\mathbf{R}^{N}} b(x)|u|^{p} d x-\frac{1}{q+1} \int_{\mathbf{R}^{N}} h(x)|u|^{q+1} d x \\
& -\frac{1}{s+1} \int_{\mathbf{R}^{N}} g(x)|u|^{s+1} d x, \quad \forall u \in E .
\end{aligned}
$$

A simple calculation shows that $I$ is well defined on $E$ and $I \in C^{1}(E, \mathbf{R})$ with

$$
\begin{aligned}
\left\langle I^{\prime}(u), \varphi\right\rangle= & \int_{\mathbf{R}^{N}} a(x, \nabla u) \cdot \nabla \varphi d x+\int_{\mathbf{R}^{N}} b(x)|u|^{p-2} u \varphi d x \\
& -\int_{\mathbf{R}^{N}} h(x)|u|^{q-1} u \varphi d x-\int_{\mathbf{R}^{N}} g(x)|u|^{s-1} u \varphi d x,
\end{aligned}
$$

for all $u$ and $\varphi \in E$.

LEMMA 5.1. The following assertions hold.

(i) There exist $\rho>0$ and $\varrho>0$ such that

$$
I(u) \geq \varrho>0, \quad \forall u \in E \text { with }\|u\|=\rho .
$$

(ii) There exists $\psi \in E$ such that

$$
\lim _{t \rightarrow \infty} I(t \psi)=-\infty
$$

(iii) There exists $\varphi \in E$ such that $\varphi \geq 0, \varphi \neq 0$ and

$$
I(t \varphi)<0
$$

for $t>0$ small enough. 
Proof. (i) First, let $\mathscr{S}$ be the best Sobolev constant of the embedding $W^{1, p}\left(\mathbf{R}^{N}\right) \hookrightarrow$ $L^{p^{\star}}\left(\mathbf{R}^{N}\right)$, that is,

$$
\mathscr{S}=\inf _{u \in W^{1, p}\left(\mathbf{R}^{N}\right) \backslash\{0\}} \frac{\int_{\mathbf{R}^{N}}|\nabla u|^{p} d x}{\left(\int_{\mathbf{R}^{N}}|u|^{p^{\star}} d x\right)^{p / p^{\star}}} .
$$

Thus we obtain

$$
\mathscr{S}^{1 / p}\|v\|_{L^{p^{\star}}\left(\mathbf{R}^{N}\right)} \leq\|v\|, \quad \forall v \in E .
$$

By Hölder's inequality and relation (5.7) we deduce

$$
\begin{aligned}
\int_{\mathbf{R}^{N}} h(x)|u|^{q^{+1}} d x & \leq\|h\|_{L^{q_{0}}\left(\mathbf{R}^{N}\right)} \cdot\|u\|_{L^{p^{\star}}\left(\mathbf{R}^{N}\right)}^{q+1} \\
& \leq\|h\|_{L^{q_{0}\left(\mathbf{R}^{N}\right)}} \cdot \frac{1}{\mathscr{S}(q+1) / p} \cdot\left(\mathscr{S}^{1 / p} \cdot\|u\|_{L^{p^{\star}}\left(\mathbf{R}^{N}\right)}\right)^{q+1} \\
& \leq\|h\|_{L^{q_{0}}\left(\mathbf{R}^{N}\right)} \cdot \frac{1}{\mathscr{S}(q+1) / p} \cdot\|u\|^{q+1} \\
& \leq(q+1) \mu\|u\|^{q+1}
\end{aligned}
$$

where $\mu=\|h\|_{L^{q_{0}\left(\mathbf{R}^{N}\right)}} /\left[(q+1) \mathscr{S}^{(q+1) / p}\right]$. With similar arguments we have

$$
\int_{\mathbf{R}^{N}} g(x)|u|^{s+1} d x \leq(p+1) v\|u\|^{s+1},
$$

where $v=\|g\|_{L^{s_{0}\left(\mathbf{R}^{N}\right)}} /\left[(p+1) \mathscr{S}^{(s+1) / p}\right]$.

Thus, we obtain

$$
\begin{aligned}
I(u) & \geq \min \left\{\Lambda, \frac{1}{p}\right\} \cdot\left\|u_{n}\right\|^{p}-\mu \cdot\|u\|^{q+1}-v \cdot\|u\|^{s+1} \\
& =\left(\lambda-\mu \cdot\|u\|^{q+1-p}-v \cdot\|u\|^{s+1-p}\right) \cdot\|u\|^{p}, \quad \forall u \in E,
\end{aligned}
$$

where $\lambda=\min \{\Lambda, 1 / p\}>0$. We show that there exists $t_{0}>0$ such that

$$
\mu \cdot t_{0}^{q+1-p}+\nu \cdot t_{0}^{s+1-p}<\lambda
$$

To do that we define the function

$$
Q(t)=\mu \cdot t^{q+1-p}+v \cdot t^{s+1-p}, \quad t>0
$$

Since $\lim _{t \rightarrow 0} Q(t)=\lim _{t \rightarrow \infty} Q(t)=\infty$ it follows that $Q$ possesses a positive minimum, say $t_{0}>0$. In order to find $t_{0}$ we have to solve equation $Q^{\prime}\left(t_{0}\right)=0$, where $Q^{\prime}(t)=(q+1-p)$. $\mu \cdot t^{q-p}+(s+1-p) \cdot v \cdot t^{s-p}$. A simple computation yields $t_{0}=[((p-q-1) /(s+1-p)) \cdot$ $(\mu / \nu)]^{1 /(s-q)}$. Thus relation $(5.11)$ holds provided that

$$
\mu \cdot\left[\frac{p-q-1}{s+1-p} \cdot \frac{\mu}{\nu}\right]^{(q+1-p) /(s-q)}+\nu \cdot\left[\frac{p-q-1}{s+1-p} \cdot \frac{\mu}{\nu}\right]^{(s+1-p) /(s-q)}<\lambda .
$$


Since $\mu=C_{1} \cdot\|h\|_{L^{q_{0}}\left(\mathbf{R}^{N}\right)}$ and $\nu=C_{2} \cdot\|g\|_{L^{s_{0}\left(\mathbf{R}^{N}\right)}}$ with $C_{1}, C_{2}$ positive constants, we deduce that (5.13) holds true if and only if the following inequality holds

$$
C_{3} \cdot\|h\|_{L^{q_{0}}\left(\mathbf{R}^{N}\right)}^{(s+1-p) /(s-q)} \cdot\|g\|_{L^{s_{0}}\left(\mathbf{R}^{N}\right)}^{(p-q-1) /(s-q)}<\lambda
$$

where $C_{3}$ is a positive constant. But inequality (5.14) holds provided that product $\|h\|_{L^{q_{0}}\left(\mathbf{R}^{N}\right)}^{(s+1-p) /(s-q)} \cdot\|g\|_{L^{s_{0}}\left(\mathbf{R}^{N}\right)}^{(p-q-1) /(s-q)}$ is small enough.

(ii) Let $\psi \in C_{0}^{\infty}\left(\mathbf{R}^{N}\right), \psi \geq 0, \psi \neq 0$. Then using relation (4.12) we have

$$
\begin{aligned}
I(t \psi)= & \int_{\mathbf{R}^{N}} A(x, t \nabla \psi) d x+\frac{t^{p}}{p} \int_{\mathbf{R}^{N}} b(x)|\psi|^{p} d x \\
& -\frac{t^{q+1}}{q+1} \int_{\mathbf{R}^{N}} h(x)|\psi|^{q+1} d x-\frac{t^{s+1}}{s+1} \int_{\mathbf{R}^{N}} g(x)|\psi|^{s+1} d x \\
\leq & t^{p} \int_{\mathbf{R}^{N}} A(x, \nabla \psi) d x+\frac{t^{p}}{p} \int_{\mathbf{R}^{N}} b(x)|\psi|^{p} d x-\frac{t^{s+1}}{s+1} \int_{\mathbf{R}^{N}} g(x)|\psi|^{s+1} d x .
\end{aligned}
$$

Thus $I(t \psi) \rightarrow-\infty$ as $t \rightarrow \infty$ and (ii) is proved.

(iii) Let $\varphi \in C_{0}^{\infty}\left(\mathbf{R}^{N}\right), \varphi \geq 0, \varphi \neq 0$ and $t>0$. Then the above inequality implies

$$
I(t \varphi) \leq t^{p} \int_{\mathbf{R}^{N}} A(x, \nabla \varphi) d x+\frac{t^{p}}{p} \int_{\mathbf{R}^{N}} b(x)|\varphi|^{p} d x-\frac{t^{q+1}}{q+1} \int_{\mathbf{R}^{N}} h(x)|\varphi|^{q+1} d x<0
$$

for $t<\delta^{1 /(p-q-1)}$ with

$$
\delta=\frac{(1 /(q+1)) \int_{\mathbf{R}^{N}} h(x)|\varphi|^{q+1} d x}{\left[\int_{\mathbf{R}^{N}} A(x, \nabla \varphi) d x+(1 / p) \int_{\mathbf{R}^{N}} b(x)|\varphi|^{p} d x\right]} .
$$

It follows that (iii) holds true.

The proof of Lemma 5.1 is complete.

Proof of Theorem 2.2. Using Lemma 5.1 and the Mountain Pass theorem we deduce the existence of a sequence $\left\{u_{n}\right\}$ in $E$ such that

$$
I\left(u_{n}\right) \longrightarrow \bar{c}>0, \quad I^{\prime}\left(u_{n}\right) \longrightarrow 0 \quad \text { in } E^{\star} .
$$

We prove that $\left\{u_{n}\right\}$ is bounded in $E$. We assume by contradiction that $\left\|u_{n}\right\| \rightarrow \infty$ as $n \rightarrow$ $\infty$. Using relation (5.18) and conditions (A4) and (A5) we deduce that for $n$ large enough we obtain

$$
\begin{aligned}
\bar{c}+1+\left\|u_{n}\right\| \geq & I\left(u_{n}\right)-\frac{1}{s+1}\left\langle I^{\prime}\left(u_{n}\right), u_{n}\right\rangle \\
= & \int_{\mathbf{R}^{N}}\left(A\left(x, \nabla u_{n}\right)-\frac{1}{s+1} a\left(x, \nabla u_{n}\right) \cdot \nabla u_{n}\right) d x \\
& +\left(\frac{1}{p}-\frac{1}{s+1}\right) \int_{\mathbf{R}^{N}} b(x)\left|u_{n}\right|^{q+1} d x \\
& -\frac{s-q}{(q+1)(s+1)} \int_{\mathbf{R}^{N}} h(x)\left|u_{n}\right|^{q+1} d x
\end{aligned}
$$


or

$$
\begin{aligned}
\bar{c}+1+ & \left\|u_{n}\right\|+\frac{s-q}{(q+1)(s+1)} \int_{\mathbf{R}^{N}} h(x)\left|u_{n}\right|^{q+1} d x \\
\geq & \left(1-\frac{p}{s+1}\right) \Lambda \int_{\mathbf{R}^{N}}\left|\nabla u_{n}\right|^{p} d x \\
& +\left(\frac{1}{p}-\frac{1}{s+1}\right) \int_{\mathbf{R}^{N}} b(x)\left|u_{n}\right|^{p} d x \\
\geq & \min \left\{\left(1-\frac{p}{s+1}\right) \Lambda,\left(\frac{1}{p}-\frac{1}{s+1}\right)\right\} \cdot\left\|u_{n}\right\|^{p} .
\end{aligned}
$$

By relation (5.8) and the above inequality we obtain

$$
\begin{aligned}
\bar{c}+1 & +\left\|u_{n}\right\|+\frac{s-q}{(q+1)(s+1)} \cdot\|h\|_{L^{q 0}\left(\mathbf{R}^{N}\right)} \cdot \frac{1}{\mathscr{S}(q+1) / p} \cdot\left\|u_{n}\right\|^{q+1} \\
& \geq \min \left\{\left(1-\frac{p}{s+1}\right) \Lambda,\left(\frac{1}{p}-\frac{1}{s+1}\right)\right\} \cdot\left\|u_{n}\right\|^{p} .
\end{aligned}
$$

Since $1<q<p-1$ and $\left\|u_{n}\right\| \rightarrow \infty$, dividing the above inequality by $\left\|u_{n}\right\|^{p}$ and passing to the limit as $n \rightarrow \infty$ we obtain a contradiction. Thus $\left\{u_{n}\right\}$ is bounded in $E$. It follows that there exists $u_{1} \in E$ such that passing to a subsequence, still denoted by $\left\{u_{n}\right\}$, it converges weakly to $u_{1}$ in $E$ and $u_{n}(x) \rightarrow u_{1}(x)$ a.e. $x \in \mathbf{R}^{N}$. With the same arguments as those used in the proof of relation (4.29) we can show that

$$
\lim _{n \rightarrow \infty}\left\langle T^{\prime}\left(u_{n}\right), u_{n}-u_{1}\right\rangle=0
$$

where $T$ is the functional defined in the third section.

Then applying Proposition 3.2 we deduce that $\left\{u_{n}\right\}$ converges strongly to $u_{1}$ in $E$. Since $I \in C^{1}(E, \mathbf{R})$ relation (5.18) implies $\left\langle I^{\prime}\left(u_{1}\right), \varphi\right\rangle=0$ for all $\varphi \in E$, that is, $u_{1}$ is a weak solution of problem (2.11). Relation (5.18) also yields $I\left(u_{1}\right)=\bar{c}>0$ and thus $u_{1}$ is non-trivial.

We prove now that there exists a second weak solution $u_{2} \in E$ such that $u_{2} \neq u_{1}$. By Lemma 5.1(i) it follows that there exists a ball centered at the origin $B \subset E$, such that

$$
\inf _{\partial B} I>0
$$

On the other hand, by Lemma 5.1(iii) there exists $\phi \in E$ such that $I(t \phi)<0$, for all $t>0$ small enough. Recalling that relation (5.10) holds for all $u \in E$, that is,

$$
I(u) \geq \lambda \cdot\|u\|^{p}-\mu \cdot\|u\|^{q+1}-v \cdot\|u\|^{s+1}
$$

we get that

$$
-\infty<\underline{c}:=\inf _{\bar{B}} I<0
$$


We let now $0<\epsilon<\inf _{\partial B} I-\inf _{B} I$. Applying Ekeland's Variational principle for functional $I: \bar{B} \rightarrow \mathbf{R}$, (see $[8]$ ), there exists $u_{\epsilon} \in \bar{B}$ such that

$$
\begin{gathered}
I\left(u_{\epsilon}\right)<\inf _{\bar{B}} I+\epsilon \\
I\left(u_{\epsilon}\right)<I(u)+\epsilon \cdot\left\|u-u_{\epsilon}\right\|, \quad u \neq u_{\epsilon} .
\end{gathered}
$$

Since

$$
I\left(u_{\epsilon}\right) \leq \inf _{\bar{B}} I+\epsilon \leq \inf _{B} I+\epsilon<\inf _{\partial B} I
$$

it follows that $u_{\epsilon} \in B$. Now, we define $M: \bar{B} \rightarrow \mathbf{R}$ by $M(u)=I(u)+\epsilon \cdot\left\|u-u_{\epsilon}\right\|$. It is clear that $u_{\epsilon}$ is a minimum point of $\mathcal{M}$ and thus

$$
\frac{M\left(u_{\epsilon}+\zeta \cdot v\right)-M\left(u_{\epsilon}\right)}{\zeta} \geq 0
$$

for a small $\zeta>0$ and $v$ in the unit sphere of $E$. The above relation yields

$$
\frac{I\left(u_{\epsilon}+\zeta \cdot v\right)-I\left(u_{\epsilon}\right)}{\zeta}+\epsilon \cdot\|v\| \geq 0
$$

Letting $\zeta \rightarrow 0$ it follows that $\left\langle I^{\prime}\left(u_{\epsilon}\right), v\right\rangle+\epsilon \cdot\|v\|>0$ and we infer that $\left\|I^{\prime}\left(u_{\epsilon}\right)\right\| \leq \epsilon$. We deduce that there exists $\left\{u_{n}\right\} \subset B$ such that $I\left(u_{n}\right) \rightarrow \underline{c}$ and $I^{\prime}\left(u_{n}\right) \rightarrow 0$. Using the same arguments as in the case of solution $u_{1}$ we can prove that $\left\{u_{n}\right\}$ converges strongly to $u_{2}$ in $E$. Moreover, that fact yields that $I^{\prime}\left(u_{2}\right)=0$. Thus, $u_{2}$ is a weak solution for (2.11) and since $0>\underline{c}=I\left(u_{2}\right)$ it follows that $u_{2}$ is non-trivial.

Finally, we point out the fact that $u_{1} \neq u_{2}$ since

$$
I\left(u_{1}\right)=\bar{c}>0>\underline{c}=I\left(u_{2}\right) .
$$

The proof of Theorem 2.2 is complete.

\section{Acknowledgment}

The author would like to thank Professor V. Rădulescu for proposing these problems and for numerous valuable discussions.

\section{References}

[1] C. O. Alves, J. V. Gonçalves, and O. H. Miyagaki, On elliptic equations in $\mathbf{R}^{N}$ with critical exponents, Electronic Journal of Differential Equations 1996 (1996), no. 9, 1-11.

[2] A. Ambrosetti and P. H. Rabinowitz, Dual variational methods in critical point theory and applications, Journal of Functional Analysis 14 (1973), no. 4, 349-381.

[3] H. Brezis, Analyse Fonctionnelle. Théorie et Applications, Collection of Applied Mathematics for the Master's Degree, Masson, Paris, 1983.

[4] P. De Nápoli and M. C. Mariani, Mountain pass solutions to equations of p-Laplacian type, Nonlinear Analysis. Theory, Methods \& Applications. An International Multidisciplinary Journal. Series A: Theory and Methods 54 (2003), no. 7, 1205-1219. 
[5] J. I. Díaz, Nonlinear Partial Differential Equations and Free Boundaries. Vol. I. Elliptic Equations, Research Notes in Mathematics, vol. 106, Pitman, Massachusetts, 1985.

[6] J. M. B. do Ó, Existence of solutions for quasilinear elliptic equations, Journal of Mathematical Analysis and Applications 207 (1997), no. 1, 104-126.

[7] Solutions to perturbed eigenvalue problems of the p-Laplacian in $\mathbf{R}^{N}$, Electronic Journal of Differential Equations 1997 (1997), no. 11, 1-15.

[8] I. Ekeland, On the variational principle, Journal of Mathematical Analysis and Applications 47 (1974), no. 2, 324-353.

[9] J. V. Gonçalves and O. H. Miyagaki, Multiple positive solutions for semilinear elliptic equations in $\mathbb{R}^{N}$ involving subcritical exponents, Nonlinear Analysis. Theory, Methods \& Applications. An International Multidisciplinary Journal. Series A: Theory and Methods 32 (1998), no. 1, 41-51.

[10] M. Mihăilescu and V. Rădulescu, Ground state solutions of non-linear singular Schrödinger equations with lack of compactness, Mathematical Methods in the Applied Sciences 26 (2003), no. 11, 897-906.

[11] D. Motreanu and V. Rădulescu, Eigenvalue problems for degenerate nonlinear elliptic equations in anisotropic media, Boundary Value Problems 2005 (2005), no. 2, 107-127.

[12] K. Pflüger, Existence and multiplicity of solutions to a p-Laplacian equation with nonlinear boundary condition, Electronic Journal of Differential Equations 1998 (1998), no. 10, 1-13.

[13] P. H. Rabinowitz, On a class of nonlinear Schrödinger equations, Zeitschrift für Angewandte Mathematik und Physik. ZAMP. Journal of Applied Mathematics and Physics. Journal de Mathématiques et de Physique Appliquées 43 (1992), no. 2, 270-291.

[14] V. Rădulescu and D. Smets, Critical singular problems on infinite cones, Nonlinear Analysis. Theory, Methods \& Applications. An International Multidisciplinary Journal. Series A: Theory and Methods 54 (2003), no. 6, 1153-1164.

[15] F. Şt. Cîrstea and V. Rădulescu, Multiple solutions of degenerate perturbed elliptic problems involving a subcritical Sobolev exponent, Topological Methods in Nonlinear Analysis 15 (2000), no. 2, 283-300.

[16] G. Tarantello, On nonhomogeneous elliptic equations involving critical Sobolev exponent, Annales de l'Institut Henri Poincaré. Analyse Non Linéaire 9 (1992), no. 3, 281-304.

[17] M. Willem, Analyse harmonique réelle, Methods Collection, Hermann, Paris, 1995.

Mihai Mihăilescu: Department of Mathematics, University of Craiova, 200585 Craiova, Romania E-mail address: mmihailes@yahoo.com 